J. Korean Math. Soc. 50 (2013), No. 5, pp. 933-944

http://dx.doi.org/10.4134/JKMS.2013.50.5.933

\title{
ON CONTINUOUS MODULE HOMOMORPHISMS BETWEEN RANDOM LOCALLY CONVEX MODULES
}

\author{
XiA ZHANG
}

\begin{abstract}
Based on the four kinds of theoretical definitions of the continuous module homomorphism between random locally convex modules, we first show that among them there are only two essentially. Further, we prove that such two are identical if the family of $L^{0}$-seminorms for the former random locally convex module has the countable concatenation property, meantime we also provide a counterexample which shows that it is necessary to require the countable concatenation property.
\end{abstract}

\section{Introduction}

The notion of random normed modules (briefly, $R N$ modules), which was first introduced by Guo in [3] and subsequently elaborated in [5], is a random generalization of that of ordinary normed spaces. Before 2009, an $R N$ module and its generalization - a random locally convex module [6] are often endowed with the $(\varepsilon, \lambda)$-topology. In fact, the $(\varepsilon, \lambda)$-topology is very natural, for example, let $L^{0}(\mathcal{F}, K)$ be the algebra of equivalence classes of random variables from a probability space $(\Omega, \mathcal{F}, P)$ to the scalar field $K$ of real or complex numbers, then $L^{0}(\mathcal{F}, K)$ is a simpler $R N$ module and the $(\varepsilon, \lambda)$-topology on $L^{0}(\mathcal{F}, K)$ is exactly the topology of convergence in probability $P$. Thus, $R N$ modules and random locally convex modules are not locally convex space in general under the $(\varepsilon, \lambda)$-topology, which also leads to the universal failure of the theory of classical conjugate spaces to serve the development of $R N$ modules, it is to overcome this obstacle that the theory of random conjugate spaces universally suitable for the development of $R N$ modules and random locally convex modules has been developed [5]. In the past ten years from 1999 to 2009, the theory of $R N$ modules and random locally convex modules has obtain some deep advances under the framework of random conjugate spaces, for example, it was

Received February 7, 2012; Revised April 12, 2013.

2010 Mathematics Subject Classification. Primary 46H25, 46A03, $46 \mathrm{E} 10$.

Key words and phrases. random locally convex modules, $(\varepsilon, \lambda)$-topology, locally $L^{0}$ convex topology, continuous module homomorphisms.

The author would like to express his sincere gratitude to Prof. Guo Tiexin for his invaluable suggestions. This research is supported by the National Natural Science Foundation of China (Grant No. 11301380 and 11226321). 
proved in [10] that the famous James theorem is always true for any complete $R N$ modules, whereas the famous determination theorem concerning the weak compactness universally fails to hold (see [7] for details).

In 2009, motivated by financial applications, D. Filipović, M. Kupper and $\mathrm{N}$. Vogelpoth presented a new topology for $R N$ modules and random locally convex modules [2], called the locally $L^{0}$-convex topology, so that the subsequent development of $R N$ modules and random locally convex modules were carried out under the framework of the two kinds of topologies. First, the principal relations between some basic results derived from the two kinds of topologies for random locally convex modules were studied in [8]. Then, based on these, lots of new and basic researches have recently been done in $[11,12,13,17,18,19,20,21,22,23]$. In this process, Guo first deeply considers the problem of applying the theory of $R N$ modules and random locally convex modules to $L^{0}$-conditional risk measures, for example, the Fenchel-Moreau dual representation theorem and the continuity and subdifferentiability theorems for $L^{0}$-convex functions were pointed out in a proper form in [9], subsequently, Guo found that the study of $L^{p}$-conditional risk measures can be incorporated into that of $L_{\mathcal{F}}^{p}(\mathcal{E})$-conditional risk measures and further established a complete random convex analysis over random locally convex modules under the two kinds of topologies in [14].

Recently, the development of the theory of random conjugate spaces has been occupying a central place in random metric theory. In particular, Guo and Zhao discussed four kinds of the random conjugate spaces of a random locally convex module under the two kinds of topologies and obtained some crucial results in [13], which motivated the author in this paper to study the precise relations among four kinds of the continuous module homomorphism between random locally convex modules. What is more important is that a counterexample is constructed in this process, which shows that the countable concatenation property is essential, and this counterexample can also be applied to random conjugate spaces.

To introduce the main results of this paper, we first recall some notation and terminology as follows. Throughout the paper, $K$ always denotes the scalar field $R$ of real numbers or $C$ of complex numbers, $(\Omega, \mathcal{F}, P)$ a probability space and $L^{0}(\mathcal{F}, K)$ the algebra of equivalence classes of $K$-valued random variables on $(\Omega, \mathcal{F}, P)$ under the ordinary addition, multiplication and scalar multiplication operations on equivalence classes.

Denote by $\bar{L}^{0}(\mathcal{F}, R)$ the set of equivalence classes of extended real-valued random variables on $(\Omega, \mathcal{F}, P)$. Then it is well known from [1] that $\bar{L}^{0}(\mathcal{F}, R)$ is partially ordered by $\xi \leq \eta$ if and only if $\xi^{0}(\omega) \leq \eta^{0}(\omega)$ for $P$-almost all $\omega$ in $\Omega$ (briefly, a.s.), where $\xi^{0}$ and $\eta^{0}$ are arbitrarily chosen representatives of $\xi$ and $\eta$ in $\bar{L}^{0}(\mathcal{F}, R)$, respectively. Furthermore, every subset $H$ of $\bar{L}^{0}(\mathcal{F}, R)$ has a supremum and infimum, denoted by $\bigvee H$ and $\bigwedge H$, respectively. It is also well known from [1] that $L^{0}(\mathcal{F}, R)$, as a sublattice of $\bar{L}^{0}(\mathcal{F}, R)$, is a complete lattice in the sense that every subset with an upper bound has a supremum. 
As usual, $\xi>\eta$ means $\xi \geq \eta$ and $\xi \neq \eta$, whereas $\xi>\eta$ on $A$ means $\xi^{0}(\omega)>\eta^{0}(\omega)$ a.s. on $A$ for any $A \in \mathcal{F}$ and $\xi$ and $\eta$ in $\bar{L}^{0}(\mathcal{F}, R)$, where $\xi^{0}$ and $\eta^{0}$ are arbitrarily chosen representatives of $\xi$ and $\eta$, respectively.

Besides, we denote $L_{+}^{0}(\mathcal{F})=\left\{\xi \in L^{0}(\mathcal{F}, R) \mid \xi \geq 0\right\}$ and $L_{++}^{0}(\mathcal{F})=\{\xi \in$ $L^{0}(\mathcal{F}, R) \mid \xi>0$ on $\left.\Omega\right\}$.

Definition $1.1([5,6,9])$. An ordered pair $(S,\|\cdot\|)$ is called an $R N$ module over $K$ with base $(\Omega, \mathcal{F}, P)$ if $S$ is a left module over the algebra $L^{0}(\mathcal{F}, K)$ and $\|\cdot\|$ is a mapping from $S$ to $L_{+}^{0}(\mathcal{F})$ such that the following three axioms are satisfied:

(1) $\|x\|=0$ if and only if $x=\theta$ (the null vector of $S$ );

(2) $\|\xi x\|=|\xi|\|x\|, \forall \xi \in L^{0}(\mathcal{F}, K)$ and $x \in S$;

(3) $\|x+y\| \leq\|x\|+\|y\|, \forall x, y \in S$,

where the mapping $\|\cdot\|$ is called the $L^{0}$-norm on $S$ and $\|x\|$ is called the $L^{0}$ norm of a vector $x \in S$. Furthermore, a mapping $\|\cdot\|: S \rightarrow L_{+}^{0}(\mathcal{F})$ satisfying (2) and (3) is called an $L^{0}$-seminorm on $S$.

Definition $1.2([6])$. An ordered pair $(S, \mathcal{P})$ is called a random locally module over $K$ with base $(\Omega, \mathcal{F}, P)$ if $S$ is a left module over the algebra $L^{0}(\mathcal{F}, K)$ and $\mathcal{P}$ is a family of mappings from $S$ to $L_{+}^{0}(\mathcal{F})$ such that the following two axioms are satisfied:

(1) Each $\|\cdot\| \in \mathcal{P}$ is an $L^{0}$-seminorm on $S$;

(2) $\bigvee\{\|x\|\|\| \cdot \| \in \mathcal{P}\}=0$ implies $x=\theta$.

In addition, it is easy to see that when $\mathcal{P}$ reduces to a singleton $\{\|\cdot\|\}$, then a random locally convex module $(S, \mathcal{P})$ is exactly an $R N$ module. Specifically, $\left(L^{0}(\mathcal{F}, K),|\cdot|\right)$ is an $R N$ module.

Given two random locally convex modules $\left(S^{1}, \mathcal{P}^{1}\right)$ and $\left(S^{2}, \mathcal{P}^{2}\right)$ over $K$ with base $(\Omega, \mathcal{F}, P)$, for the sake of convenience, we always denote the $(\varepsilon, \lambda)$-topology and the locally $L^{0}$-convex topology for $\left(S^{i}, \mathcal{P}^{i}\right)$ by $\mathcal{T}_{\varepsilon, \lambda}$ and $\mathcal{T}_{c}(i=1,2)$, respectively (see $[2,8]$ and also Section 2 for the definitions of two kinds of topologies). It should be pointed out that $\left(L^{0}(\mathcal{F}, K), \mathcal{T}_{\varepsilon, \lambda}\right)$ is a topological algebra, whereas $\left(L^{0}(\mathcal{F}, K), \mathcal{T}_{c}\right)$ is only a topological ring (see [8] for details).

We can now introduce Definition 1.3 below.

Definition 1.3. Let $\left(S^{1}, \mathcal{P}^{1}\right)$ and $\left(S^{2}, \mathcal{P}^{2}\right)$ be two random locally convex modules over $K$ with base $(\Omega, \mathcal{F}, P)$ and define $B\left(S_{\varepsilon, \lambda}^{1}, S_{\varepsilon, \lambda}^{2}\right), B\left(S_{c}^{1}, S_{c}^{2}\right), B\left(S_{c}^{1}, S_{\varepsilon, \lambda}^{2}\right)$ and $B\left(S_{\varepsilon, \lambda}^{1}, S_{c}^{2}\right)$ as follows:

(1) $B\left(S_{\varepsilon, \lambda}^{1}, S_{\varepsilon, \lambda}^{2}\right)=\{T \mid T$ is a continuous module homomorphism from $\left(S^{1}, \mathcal{T}_{\varepsilon, \lambda}\right)$ to $\left.\left(S^{2}, \mathcal{T}_{\varepsilon, \lambda}\right)\right\}$

(2) $B\left(S_{c}^{1}, S_{c}^{2}\right)=\left\{T \mid T\right.$ is a continuous module homomorphism from $\left(S^{1}, \mathcal{T}_{c}\right)$ to $\left.\left(S^{2}, \mathcal{T}_{c}\right)\right\}$;

(3) $B\left(S_{c}^{1}, S_{\varepsilon, \lambda}^{2}\right)=\{T \mid T$ is a continuous module homomorphism from $\left(S^{1}, \mathcal{T}_{c}\right)$ to $\left.\left(S^{2}, \mathcal{T}_{\varepsilon, \lambda}\right)\right\}$, where $\left(S^{2}, \mathcal{T}_{\varepsilon, \lambda}\right)$ is viewed as a topological module over the topological ring $\left(L^{0}(\mathcal{F}, K), \mathcal{T}_{\varepsilon, \lambda}\right)$; 
(4) $B\left(S_{\varepsilon, \lambda}^{1}, S_{c}^{2}\right)=\{T \mid T$ is a continuous module homomorphism from $\left(S^{1}, \mathcal{T}_{\varepsilon, \lambda}\right)$ to $\left.\left(S^{2}, \mathcal{T}_{c}\right)\right\}$, where $\left(S^{1}, \mathcal{T}_{\varepsilon, \lambda}\right)$ is viewed as a topological module over the topological ring $\left(L^{0}(\mathcal{F}, K), \mathcal{T}_{\varepsilon, \lambda}\right)$.

Since the locally $L^{0}$-convex topology of random locally convex modules is much stronger than its $(\varepsilon, \lambda)$-topology, one can obtain that

$$
B\left(S_{\varepsilon, \lambda}^{1}, S_{c}^{2}\right) \subseteq B\left(S_{c}^{1}, S_{c}^{2}\right) \subseteq B\left(S_{\varepsilon, \lambda}^{1}, S_{\varepsilon, \lambda}^{2}\right) \subseteq B\left(S_{c}^{1}, S_{\varepsilon, \lambda}^{2}\right) .
$$

In particular, if we take $\left(S^{2}, \mathcal{P}^{2}\right)=\left(L^{0}(\mathcal{F}, K),|\cdot|\right)$, then $B\left(S_{\varepsilon, \lambda}^{1}, S_{\varepsilon, \lambda}^{2}\right), B\left(S_{c}^{1}, S_{c}^{2}\right)$, $B\left(S_{c}^{1}, S_{\varepsilon, \lambda}^{2}\right)$ and $B\left(S_{\varepsilon, \lambda}^{1}, S_{c}^{2}\right)$ are exactly $\left(S_{\varepsilon, \lambda}^{1}\right)^{*},\left(S_{c}^{1}\right)^{*},\left(S_{\max }^{1}\right)^{*}$ and $\left(S_{\min }^{1}\right)^{*}$ in [13]. In fact, it is the relations among $\left(S_{\varepsilon, \lambda}^{1}\right)^{*},\left(S_{c}^{1}\right)^{*},\left(S_{\max }^{1}\right)^{*}$ and $\left(S_{\min }^{1}\right)^{*}$ that motivate the author in this paper to study the relations among the above four kinds of continuous module homomorphisms.

Based on the above preliminaries, we present the main results of this paper as follows:

Theorem 1.4. Let $\left(S^{1}, \mathcal{P}^{1}\right)$ and $\left(S^{2}, \mathcal{P}^{2}\right)$ be two random locally convex modules over $K$ with base $(\Omega, \mathcal{F}, P)$ and $T \in B\left(S_{\varepsilon, \lambda}^{1}, S_{c}^{2}\right)$. If $(\Omega, \mathcal{F}, P)$ is nonatomic, then $T x=\theta, \forall x \in S^{1}$, namely $B\left(S_{\varepsilon, \lambda}^{1}, S_{c}^{2}\right)=\{\theta\}$.

Theorem 1.5. Let $\left(S^{1}, \mathcal{P}^{1}\right)$ and $\left(S^{2}, \mathcal{P}^{2}\right)$ be two random locally convex modules over $K$ with base $(\Omega, \mathcal{F}, P)$. If $\mathcal{P}^{1}$ has the countable concatenation property, then $B\left(S_{c}^{1}, S_{c}^{2}\right)=B\left(S_{\varepsilon, \lambda}^{1}, S_{\varepsilon, \lambda}^{2}\right)$.

Theorem 1.6. Let $\left(S^{1}, \mathcal{P}^{1}\right)$ and $\left(S^{2}, \mathcal{P}^{2}\right)$ be two random locally convex modules over $K$ with base $(\Omega, \mathcal{F}, P)$. Then $B\left(S_{\varepsilon, \lambda}^{1}, S_{\varepsilon, \lambda}^{2}\right)=B\left(S_{c}^{1}, S_{\varepsilon, \lambda}^{2}\right)$.

It follows from Theorems 1.4 and 1.6 above that among the four kinds of continuous module homomorphisms only $B\left(S_{\varepsilon, \lambda}^{1}, S_{\varepsilon, \lambda}^{2}\right)$ and $B\left(S_{c}^{1}, S_{c}^{2}\right)$ are universally suitable for the current development of the theory of random locally convex modules, and Theorem 1.5 shows that they are identical if $\mathcal{P}^{1}$ has the countable concatenation property. It is noteworthy that a counterexample is constructed in Section 3 of this paper, which shows that Theorem 1.5 may be not true if $\mathcal{P}^{1}$ does not have such property.

\section{Two kinds of topologies}

For later use in Section 3 of this paper, let us briefly recall some notions and facts about the $(\varepsilon, \lambda)$-topology and locally $L^{0}$-convex topology for random locally convex modules in this section.

In the sequel, for a random locally convex module $(S, \mathcal{P})$ and for any finite subfamily $\mathcal{Q} \subset \mathcal{P},\|\cdot\|_{\mathcal{Q}}$ always denotes the $L^{0}$-seminorm defined by

$$
\|x\|_{\mathcal{Q}}=\bigvee\{\|x\| \mid\|\cdot\| \in \mathcal{Q}\}, \forall x \in S,
$$

unless stated otherwise. 
The $(\varepsilon, \lambda)$-topology, which is inherited from B. Schweizer and A. Sklar's work in 1960, has a long history [16]. Motivated by the above work, Guo introduced the $(\varepsilon, \lambda)$-topology for a random locally convex module.

Proposition $2.1([6])$. Let $(S, \mathcal{P})$ be a random locally convex module over $K$ with base $(\Omega, \mathcal{F}, P)$. For any real numbers $\varepsilon>0,0<\lambda<1$ and any finite subfamily $Q$ of $\mathcal{P}$, let

$$
N_{\theta}(Q, \varepsilon, \lambda)=\left\{x \in S \mid P\left\{\omega \in \Omega \mid\|x\|_{Q}(\omega)<\varepsilon\right\}>1-\lambda\right\}
$$

and

$$
\mathcal{U}_{\theta}=\left\{N_{\theta}(Q, \varepsilon, \lambda) \mid Q \subset \mathcal{P} \text { finite, } \varepsilon>0,0<\lambda<1\right\},
$$

then $\mathcal{U}_{\theta}$ is a local base at $\theta$ of some Hausdorff linear topology, called the $(\varepsilon, \lambda)$ topology induced by $\mathcal{P}$. Further, we have the following statements:

(1) $L^{0}(\mathcal{F}, K)$ is a topological algebra over $K$ endowed with its $(\varepsilon, \lambda)$-topology, which is exactly the topology of convergence in probability $P$;

(2) $S$ is a topological module over the topological algebra $L^{0}(\mathcal{F}, K)$ when $S$ and $L^{0}(\mathcal{F}, K)$ are endowed with their respective $(\varepsilon, \lambda)$-topologies;

(3) A net $\left\{x_{\alpha}, \alpha \in \wedge\right\}$ in $S$ converges in the $(\varepsilon, \lambda)$-topology to $x \in S$ if and only if $\left\{\left\|x_{\alpha}-x\right\|, \alpha \in \wedge\right\}$ converges in probability $P$ to 0 for each $\|\cdot\| \in \mathcal{P}$.

Proposition $2.2([2,8])$. Let $(S, \mathcal{P})$ be a random locally convex module over $K$ with base $(\Omega, \mathcal{F}, P)$. For any $\varepsilon \in L_{++}^{0}(\mathcal{F})$ and $Q \subset \mathcal{P}$ finite, let

$$
N_{\theta}(Q, \varepsilon)=\left\{x \in S \mid\|x\|_{Q} \leq \varepsilon\right\}
$$

and

$$
\mathcal{U}_{\theta}=\left\{N_{\theta}(Q, \varepsilon) \mid Q \subset \mathcal{P} \text { finite, } \varepsilon \in L_{++}^{0}(\mathcal{F})\right\} .
$$

$A$ set $G \subset S$ is called $\mathcal{T}_{c}$-open if for every $x \in G$ there exists some $N_{\theta}(Q, \varepsilon) \in \mathcal{U}_{\theta}$ such that $x+N_{\theta}(Q, \varepsilon) \subset G$. Let $\mathcal{T}_{c}$ be the family of $\mathcal{T}_{c}$-open subsets, then $\mathcal{T}_{c}$ is a Hausdorff topology on $S$, called the locally $L^{0}$-convex topology induced by $\mathcal{P}$. Further, the following statements are true:

(1) $L^{0}(\mathcal{F}, K)$ is a topological ring endowed with its locally $L^{0}$-convex topology;

(2) $S$ is a topological module over the topological ring $L^{0}(\mathcal{F}, K)$ when $S$ and $L^{0}(\mathcal{F}, K)$ are endowed with their respective locally $L^{0}$-convex topologies;

(3) A net $\left\{x_{\alpha}, \alpha \in \wedge\right\}$ in $S$ converges in the locally $L^{0}$-convex topology to $x \in S$ if and only if $\left\{\left\|x_{\alpha}-x\right\|, \alpha \in \wedge\right\}$ converges in the locally $L^{0}$-convex topology of $L^{0}(\mathcal{F}, K)$ to $\theta$ for each $\|\cdot\| \in \mathcal{P}$.

Since $\mathcal{T}_{c}$ is not necessarily a linear topology as proved in [8], but $\left(S, \mathcal{T}_{c}\right)$ is always a topological group with respect to the addition operation for any random locally convex module $(S, \mathcal{P})$, and hence $\mathcal{T}_{c}$-Cauchy nets and $\mathcal{T}_{c}$-completeness are still well defined. It should be pointed out that $\mathcal{T}_{c}$ is called locally $L^{0}$ convex because it has a striking local base $\mathcal{U}_{\theta}=\left\{N_{\theta}(Q, \varepsilon) \mid Q \subset \mathcal{P}\right.$ finite and $\left.\varepsilon \in L_{++}^{0}\right\}$, each member $U$ of which is: 
(i) $L^{0}$-convex: $\xi \cdot x+(1-\xi) \cdot y \in U$ for any $x, y \in U$ and $\xi \in L_{+}^{0}$ such that $0 \leq \xi \leq 1$

(ii) $L^{0}$-absorbent: there is $\xi \in L_{++}^{0}$ for each $x \in S$ such that $x \in \xi \cdot U$;

(iii) $L^{0}$-balanced: $\xi \cdot x \in U$ for any $x \in U$ and any $\xi \in L^{0}(\mathcal{F}, K)$ such that $|\xi| \leq 1$.

Remark 2.1. As shown in $[8,9]$, the $(\varepsilon, \lambda)$-topology and the locally $L^{0}$-convex topology have their respective advantages and disadvantages and they can complement each other in the study of random locally convex modules.

\section{Proof of main results}

\subsection{Proof of Theorem 1.4}

Let $S$ be a left module over that algebra $L^{0}(\mathcal{F}, K)$, a module homomorphism $f: S \rightarrow L^{0}(\mathcal{F}, K)$ is called an $L^{0}$-linear function. In order to give the proof of Theorem 1.4, let us recall Proposition 3.1 below.

Proposition $3.1([8])$. Let $S$ be a left module over that algebra $L^{0}(\mathcal{F}, K)$, $M \subset S$ an $L^{0}(\mathcal{F}, K)$-submodule, $f: M \rightarrow L^{0}(\mathcal{F}, K)$ is an $L^{0}$-linear function and $p: S \rightarrow L^{0}(\mathcal{F}, K)$ an $L^{0}$-seminorm such that $|f(x)| \leq p(x), \forall x \in M$. Then there exists an $L^{0}$-linear function $g: S \rightarrow L^{0}(\mathcal{F}, K)$ such that $g$ extends $f$ and $|g(x)| \leq p(x), \forall x \in S$.

In [13], Guo and Zhao obtained an important result, i.e., Lemma 3.1 below, which plays a key role in the proof of Theorem 1.4. Now let us recall the notion of an atom. Let $(\Omega, \mathcal{F}, P)$ be a probability space, a set $A \in \mathcal{F}$ with $P(A)>0$ is called an atom if $B \in \mathcal{F}$ and $B \subset A$, then either $P(B)=0$ or $P(A \backslash B)=0$. Clearly, if $A_{1}$ and $A_{2}$ are atoms, then either $P\left(A_{1} \cap A_{2}\right)=0$ or $P\left(A_{1} \triangle A_{2}\right)=0$. A probability space without any atoms is called nonatomic.

Lemma $3.1([13])$. Let $\left(S^{1}, \mathcal{P}^{1}\right)$ be a random locally convex module over $K$ with base $(\Omega, \mathcal{F}, P)$ and $f \in\left(S_{\min }^{1}\right)^{*}$. If $(\Omega, \mathcal{F}, P)$ is nonatomic, then $f(x)=0$, $\forall x \in S^{1}$.

We can now prove Theorem 1.4.

Proof of Theorem 1.4. For any $T \in B\left(S_{\varepsilon, \lambda}^{1}, S_{c}^{2}\right)$ and $f \in\left(S_{c}^{2}\right)^{*}$, then $f \circ T \in$ $\left(S_{\text {min }}^{1}\right)^{*}$. Since $(\Omega, \mathcal{F}, P)$ is nonatomic, it follows from Lemma 3.1 that

$$
(f \circ T)(x)=f(T x)=0, \forall x \in S^{1} .
$$

We can assert that $T x=\theta, \forall x \in S^{1}$. In fact, if not, there exists some $x_{0} \in S^{1}$ such that

$$
P\left[\bigvee\left\{\left\|T x_{0}\right\|\|\| \cdot \| \in \mathcal{P}^{2}\right\}=0\right]<1
$$

namely there exists some $\|\cdot\|_{0} \in \mathcal{P}^{2}$ such that

$$
P\left[\left\|T x_{0}\right\|_{0}>0\right]>0 .
$$


Let

$$
M=\left\{\xi \cdot T x_{0} \mid \xi \in L^{0}(\mathcal{F}, K)\right\},
$$

then $M \subset S^{2}$ and $M$ is an $L^{0}(\mathcal{F}, K)$-submodule of $S^{2}$.

Let

$$
\tilde{f}\left(\xi \cdot T x_{0}\right)=\xi \cdot\left\|T x_{0}\right\|_{0}, \forall \xi \in L^{0}(\mathcal{F}, K),
$$

then $\tilde{f}: M \rightarrow L^{0}(\mathcal{F}, K)$ is an $L^{0}$-linear function and

$$
\left|\tilde{f}\left(\xi \cdot T x_{0}\right)\right|=|\xi| \cdot\left\|T x_{0}\right\|_{0}=\left\|\xi \cdot T x_{0}\right\|_{0} .
$$

Define the mapping $p: S^{2} \rightarrow L^{0}(\mathcal{F}, K)$ by $p(x)=\|x\|_{0}$ for any $x \in S^{2}$. It is clear that $p$ is an $L^{0}$-seminorm and $|\tilde{f}(y)| \leq p(y)$ for any $y \in M$. Then it follows from Proposition 3.1 that there exists an $L^{0}$-linear function $g: S \rightarrow L^{0}(\mathcal{F}, K)$ such that $g$ extends $f$ and $|g(x)| \leq p(x), \forall x \in S$. Consequently, $g \in\left(S_{c}^{2}\right)^{*}$ and $g\left(T x_{0}\right)=\tilde{f}\left(T x_{0}\right)=\left\|T x_{0}\right\|_{0}$, which is in contradiction to the equation (3.1).

This completes the proof.

\subsection{The relation between $B\left(S_{c}^{1}, S_{c}^{2}\right)$ and $B\left(S_{\varepsilon, \lambda}^{1}, S_{\varepsilon, \lambda}^{2}\right)$}

In order to give the relation between $B\left(S_{c}^{1}, S_{c}^{2}\right)$ and $B\left(S_{\varepsilon, \lambda}^{1}, S_{\varepsilon, \lambda}^{2}\right)$, we first introduce the notion of a.s. bounded random linear operator of type I from $\left(S^{1}, \mathcal{P}^{2}\right)$ to $\left(S^{2}, \mathcal{P}^{2}\right)$ and give a characterization of each element in $B\left(S_{c}^{1}, S_{c}^{2}\right)$.

Definition 3.1. Let $\left(S^{1}, \mathcal{P}^{1}\right)$ and $\left(S^{2}, \mathcal{P}^{2}\right)$ be two random locally convex modules over $K$ with base $(\Omega, \mathcal{F}, P)$. A linear operator $T:\left(S^{1}, \mathcal{P}^{1}\right) \rightarrow\left(S^{2}, \mathcal{P}^{2}\right)$ is called an a.s. bounded random linear operator of type I if for each $\mathcal{Q}^{2} \in \mathcal{F}\left(\mathcal{P}^{2}\right)$, there exist some $\xi \in L_{+}^{0}(\mathcal{F})$ and $\mathcal{Q}^{1} \in \mathcal{F}\left(\mathcal{P}^{1}\right)$ such that

$$
\|T x\|_{\mathcal{Q}^{2}} \leq \xi \cdot\|x\|_{\mathcal{Q}^{1}}, \forall x \in S^{1},
$$

where $\mathcal{F}\left(\mathcal{P}^{1}\right)$ and $\mathcal{F}\left(\mathcal{P}^{2}\right)$ denote the sets of finite subfamilies of $\mathcal{P}^{1}$ and $\mathcal{P}^{2}$, respectively.

In this paper we distinguish random variables from their equivalence classes by means of symbols: for example, $I_{A}$ denotes the characteristic function of the $\mathcal{F}$-measurable set $A$, then we use $\tilde{I}_{A}$ for its equivalence class. Further, let $A=\left\{\omega \in \Omega \mid \xi^{0}(\omega)>\eta^{0}(\omega)\right\}$, where $\xi^{0}$ and $\eta^{0}$ are arbitrarily chosen representatives of $\xi$ and $\eta$ in $L^{0}(\mathcal{F}, R)$, respectively, then we always use [ $\xi>$ $\eta]$ for the equivalence class of $A$ and often write $I_{[\xi>\eta]}$ for $\tilde{I}_{A}$, one can also understand such notations as $I_{[\xi \leq \eta]}, I_{[\xi \neq \eta]}$ and $I_{[\xi=\eta]}$. Besides, for any $\xi \in$ $L^{0}(\mathcal{F}, K), \xi^{-1}$ stands for the equivalence class of the $\mathcal{F}$-measurable function $\left(\xi^{0}\right)^{-1}: \Omega \rightarrow K$ defined by

$$
\left(\xi^{0}\right)^{-1}(\omega)= \begin{cases}\left(\xi^{0}(\omega)\right)^{-1}, & \text { if } \xi^{0}(\omega) \neq 0 \\ 0, & \text { otherwise }\end{cases}
$$

where $\xi^{0}$ is an arbitrarily chosen representative of $\xi$. It is clear that $\xi \cdot \xi^{-1}=$ $I_{[|\xi|>0]} \cdot$ 
Similar to the proof of [15, Lemma 2.1] and [4, Theorem 2.4], one can obtain Proposition 3.2 below, which will be used in the proof of Lemma 3.2 in this paper.

Proposition 3.2. Let $\left(S^{1}, \mathcal{P}^{1}\right)$ and $\left(S^{2}, \mathcal{P}^{2}\right)$ be two random locally convex modules over $K$ with base $(\Omega, \mathcal{F}, P)$. If $T:\left(S^{1}, \mathcal{P}^{1}\right) \rightarrow\left(S^{2}, \mathcal{P}^{2}\right)$ is an a.s. bounded random linear operator of type $I$, then $T$ is a module homomorphism.

Lemma 3.2. Let $\left(S^{1}, \mathcal{P}^{1}\right)$ and $\left(S^{2}, \mathcal{P}^{2}\right)$ be two random locally convex modules over $K$ with base $(\Omega, \mathcal{F}, P)$. Then $T \in B\left(S_{c}^{1}, S_{c}^{2}\right)$ if and only if $T$ is an a.s. bounded random linear operator of type I from $\left(S^{1}, \mathcal{P}^{1}\right)$ to $\left(S^{2}, \mathcal{P}^{2}\right)$.

Proof. Sufficiency. It is clear according to Proposition 3.2.

Necessity. Let $T$ be a continuous module homomorphism from $\left(S^{1}, \mathcal{T}_{c}^{1}\right)$ to $\left(S^{2}, \mathcal{T}_{c}^{2}\right)$, then $T \theta=\theta$. For each $\mathcal{Q}^{2} \in \mathcal{F}\left(\mathcal{P}^{2}\right)$, let

$$
N_{\theta}\left(\mathcal{Q}^{2}, 1\right)=\left\{x \in S^{2} \mid\|x\|_{\mathcal{Q}^{2}} \leq 1\right\},
$$

then $N_{\theta}\left(\mathcal{Q}^{2}, 1\right)$ is a neighborhood of $\theta$ in $\left(S^{2}, \mathcal{T}_{c}^{2}\right)$. Since $T$ is continuous, for each $\mathcal{Q}^{2} \in \mathcal{F}\left(\mathcal{P}^{2}\right)$, it follows that there exists a neighborhood $U$ of $\theta$ in $\left(S^{1}, \mathcal{T}_{c}^{1}\right)$ such that $T x \in N_{\theta}\left(\mathcal{Q}^{2}, 1\right)$ for any $x \in U$. Moreover, there exist some $\mathcal{Q}^{1} \in \mathcal{F}\left(\mathcal{P}^{1}\right)$ and $\varepsilon \in L_{++}^{0}(\mathcal{F})$ such that $N_{\theta}\left(\mathcal{Q}^{1}, \varepsilon\right) \subset U$.

For any $x \in S^{1}$, then $\|x\|_{\mathcal{Q}^{1}} \in L_{+}^{0}(\mathcal{F})$. In the following we will divide the proof into two parts.

Case I: If $P\left[\|x\|_{\mathcal{Q}^{1}}=0\right]=0$, namely $\|x\|_{\mathcal{Q}^{1}} \in L_{++}^{0}(\mathcal{F})$, take $x_{1}=\frac{\varepsilon}{2\|x\|_{\mathcal{Q}^{1}}} x$, then

$$
\left\|x_{1}\right\|_{\mathcal{Q}^{1}}=\frac{\varepsilon}{2\|x\|_{\mathcal{Q}^{1}}} \cdot\|x\|_{\mathcal{Q}^{1}} \leq \varepsilon,
$$

namely $x_{1} \in N_{\theta}\left(\mathcal{Q}^{1}, \varepsilon\right) \subset U$, thus $T x_{1} \in N_{\theta}\left(\mathcal{Q}^{2}, 1\right)$. Since it is easy to see that $x=\frac{2\|x\|_{\mathcal{Q}^{1}}}{\varepsilon} x_{1}$, it follows that

$$
\|T x\|_{\mathcal{Q}^{2}}=\left\|\frac{2\|x\|_{\mathcal{Q}^{1}}}{\varepsilon} T x_{1}\right\|_{\mathcal{Q}^{2}}=\frac{2}{\varepsilon} \cdot\|x\|_{\mathcal{Q}^{1}} \cdot\left\|T x_{1}\right\|_{\mathcal{Q}^{2}} \leq \frac{2}{\varepsilon} \cdot\|x\|_{\mathcal{Q}^{1}} .
$$

Let $\xi=\frac{2}{\varepsilon} \in L_{++}^{0}(\mathcal{F})$. Then

$$
\|T x\|_{\mathcal{Q}^{2}} \leq \xi \cdot\|x\|_{\mathcal{Q}^{1}}
$$

Case II: If $P\left[\|x\|_{\mathcal{Q}^{1}}=0\right]>0$, let $D=\left[\|x\|_{\mathcal{Q}^{1}}=0\right]$, then $D \in \mathcal{F}$ and $P(D)>0$. Now let us consider the restraint $I_{D} \cdot\|\cdot\|_{\mathcal{Q}^{1}}$ of $\|\cdot\|_{\mathcal{Q}^{1}}$ onto $D$. For any $\lambda \in L_{++}^{0}(\mathcal{F})$, then $I_{D} \cdot\|\lambda x\|_{\mathcal{Q}^{1}}=\lambda I_{D} \cdot\|x\|_{\mathcal{Q}^{1}}=0$, thus $I_{D} \cdot \lambda x \in N_{\theta}\left(\mathcal{Q}^{1}, \varepsilon\right) \subset U$. Hence $I_{D} \cdot \lambda T x=T\left(I_{D} \cdot \lambda x\right) \in N_{\theta}\left(\mathcal{Q}^{2}, 1\right)$, namely,

$$
\left\|I_{D} \cdot \lambda T x\right\|_{\mathcal{Q}^{2}}=I_{D} \cdot \lambda\|T x\|_{\mathcal{Q}^{2}} \leq 1 .
$$

Consequently,

$$
\lambda^{0}(\omega)\|T x\|_{\mathcal{Q}^{2}}^{0}(\omega) \leq 1, \forall \omega \in D
$$

where $\lambda^{0}$ and $\|T x\|_{\mathcal{Q}^{2}}^{0}$ are arbitrary chosen representatives of $\lambda$ and $\|T x\|_{\mathcal{Q}^{2}}$, respectively. Since $\lambda$ is arbitrary, we can assert that $I_{D} \cdot\|T x\|_{\mathcal{Q}^{2}}=0$. In 
fact, if not, there exists some $D^{\prime} \in \mathcal{F}, D^{\prime} \subset D$ and $P\left(D^{\prime}\right)>0$ such that $I_{D^{\prime}} \cdot\|T x\|_{\mathcal{Q}^{2}}+I_{\left(D^{\prime}\right)^{c}} \in L_{++}^{0}(\mathcal{F})$, let

$$
\lambda=I_{D^{\prime}} \cdot \frac{2}{\|T x\|_{\mathcal{Q}^{2}}}+I_{\left(D^{\prime}\right)^{c}},
$$

then $\lambda \in L_{++}^{0}$ and

$$
\lambda^{0}(\omega)\|T x\|_{\mathcal{Q}^{2}}^{0}(\omega)=2, \forall \omega \in D^{\prime} \subset D,
$$

which is contradict to the inequality (3.2). Thus $I_{D} \cdot\|T x\|_{\mathcal{Q}^{2}}=0$.

Since $D=\left[\|x\|_{\mathcal{Q}^{1}}=0\right]$, we have $\left\|I_{D^{c}} \cdot x\right\|_{\mathcal{Q}^{1}}+I_{D} \in L_{++}^{0}(\mathcal{F})$. Thus it follows from the condition of Case I that there exists some $\xi \in L_{++}^{0}(\mathcal{F})$ such that

$$
\left\|T\left(I_{D^{c}} \cdot x\right)\right\|_{\mathcal{Q}^{2}} \leq \xi \cdot\left\|I_{D^{c}} \cdot x\right\|_{\mathcal{Q}^{1}} .
$$

Consequently,

$$
\begin{aligned}
\|T x\|_{\mathcal{Q}^{2}} & =\left\|I_{D} \cdot T x+I_{D^{c}} \cdot T x\right\|_{\mathcal{Q}^{2}} \\
& \leq\left\|I_{D} \cdot T x\right\|_{\mathcal{Q}^{2}}+\left\|I_{D^{c}} \cdot T x\right\|_{\mathcal{Q}^{2}} \\
& =\left\|I_{D^{c}} \cdot T x\right\|_{\mathcal{Q}^{2}} \\
& \leq \xi \cdot\left\|I_{D^{c}} \cdot x\right\|_{\mathcal{Q}^{1}} \\
& \leq \xi \cdot\|x\|_{\mathcal{Q}^{1}},
\end{aligned}
$$

which says that $T$ is an a.s. bounded random linear operator of type I.

This completes the proof.

Remark 3.1. Guo gave a characterization of each element in $\left(S_{c}\right)^{*}$ by a concise way of thinking in [8], following this idea, one can also give a proof for the necessity of Lemma 3.2, please refer to [8, page 3032].

Before giving the proof of Theorem 1.5, let us recall the notion of a.s. bounded random linear operator of type II from $\left(S^{1}, \mathcal{P}^{1}\right)$ to $\left(S^{2}, \mathcal{P}^{2}\right)$ and a characterization of each element in $B\left(S_{\varepsilon, \lambda}^{1}, S_{\varepsilon, \lambda}^{2}\right)$.

Definition $3.2([15])$. Let $\left(S^{1}, \mathcal{P}^{1}\right)$ and $\left(S^{2}, \mathcal{P}^{2}\right)$ be two random locally convex modules over $K$ with base $(\Omega, \mathcal{F}, P)$. A linear operator $T:\left(S^{1}, \mathcal{P}^{1}\right) \rightarrow\left(S^{2}, \mathcal{P}^{2}\right)$ is called a.s. bounded random linear operator of type II if for each $\mathcal{Q}^{2} \in \mathcal{F}\left(\mathcal{P}^{2}\right)$, there exist a countable partition $\left\{A_{n}, n \in N\right\}$ of $\Omega$ to $\mathcal{F}$, a sequence $\left\{\xi_{n}, n \in\right.$ $N\}$ in $L_{+}^{0}(\mathcal{F})$ and a countable subset $\left\{\mathcal{Q}_{n}^{1}, n \in N\right\}$ in $\mathcal{F}\left(\mathcal{P}^{1}\right)$ such that

$$
\|T x\|_{\mathcal{Q}^{2}} \leq \sum_{n=1}^{\infty} \tilde{I}_{A_{n}} \cdot \xi_{n} \cdot\|x\|_{\mathcal{Q}_{n}^{1}}, \forall x \in S^{1},
$$

where $\mathcal{F}\left(\mathcal{P}^{1}\right)$ and $\mathcal{F}\left(\mathcal{P}^{2}\right)$ are the same as in Definition 3.1.

Proposition $3.3([15])$. Let $\left(S^{1}, \mathcal{P}^{1}\right)$ and $\left(S^{2}, \mathcal{P}^{2}\right)$ be two random locally convex modules over $K$ with base $(\Omega, \mathcal{F}, P)$. Then $T \in B\left(S_{\varepsilon, \lambda}^{1}, S_{\varepsilon, \lambda}^{2}\right)$ if and only if $T:\left(S^{1}, \mathcal{P}^{1}\right) \rightarrow\left(S^{2}, \mathcal{P}^{2}\right)$ is an a.s. bounded random linear operator of type II. 
We can now prove Theorem 1.5.

Proof of Theorem 1.5. If $T \in B\left(S_{\varepsilon, \lambda}^{1}, S_{\varepsilon, \lambda}^{2}\right)$, then it follows from Proposition 3.3 that for each $\mathcal{Q}^{2} \in \mathcal{F}\left(\mathcal{P}^{2}\right)$, there exist a countable partition $\left\{A_{n}, n \in N\right\}$ of $\Omega$ to $\mathcal{F}$, a sequence $\left\{\xi_{n}, n \in N\right\}$ in $L_{+}^{0}(\mathcal{F})$ and a countable subset $\left\{\mathcal{Q}_{n}^{1}, n \in N\right\}$ in $\mathcal{F}\left(\mathcal{P}^{1}\right)$ such that

$$
\|T x\|_{\mathcal{Q}^{2}} \leq \sum_{n=1}^{\infty} \tilde{I}_{A_{n}} \cdot \xi_{n} \cdot\|x\|_{\mathcal{Q}_{n}^{1}}, \forall x \in S^{1} .
$$

Let $\xi=\sum_{n=1}^{\infty} \tilde{I}_{A_{n}} \cdot \xi_{n}$, then $\xi \in L_{+}^{0}(\mathcal{F})$. Since $\mathcal{P}^{1}$ has the countable concatenation property, it follows that there exists a $\mathcal{Q}^{1} \in \mathcal{F}\left(\mathcal{P}^{1}\right)$ such that $\|x\|_{\mathcal{Q}^{1}}=\sum_{n=1}^{\infty} \tilde{I}_{A_{n}} \cdot\|x\|_{\mathcal{Q}_{n}^{1}}$ for any $x \in S^{1}$. Thus the inequality (3.4) yields that

$$
\|T x\|_{\mathcal{Q}^{2}} \leq \xi \cdot\|x\|_{\mathcal{Q}^{1}}, \forall x \in S^{1},
$$

which shows that $T \in B\left(S_{c}^{1}, S_{c}^{2}\right)$ according to Lemma 3.2.

This completes the proof.

Now we give Example 3.1 below, which shows that it is necessary to require that $\mathcal{P}^{1}$ have the countable concatenation property in Theorem 1.5.

Example 3.1. Let $\Omega=[0,1), \mathcal{F}=$ the $\sigma$-algebra generated by the sets $A_{n}:=$ $\left[1-2^{-(n-1)}, 1-2^{-n}\right]$ for each $n \in N$ and $P=$ the Lebesgue measure on $[0,1)$. Then clearly $(\Omega, \mathcal{F}, P)$ is a probability space. Let $S=L^{0}(\mathcal{F}, R)$ and $|\cdot|=$ the ordinary $L^{0}$-norm on $S$. For each $n \in N$, define the mapping $|\cdot|_{n}: S \rightarrow L_{+}^{0}(\mathcal{F})$ by

$$
|x|_{n}=\tilde{I}_{A_{n}} \cdot|x|, \forall x \in S,
$$

then each $|\cdot|_{n}$ is an $L^{0}$-seminorm on $S$. Let $\mathcal{P}^{1}=\left\{|\cdot|_{n} \mid n \in N\right\}$, then it is easy to see that

$$
|\cdot|=\sum_{n=1}^{\infty}|\cdot|_{n} \notin \mathcal{P}^{1},
$$

that is to say, $\left(S, \mathcal{P}^{1}\right)$ is a random locally convex module over $R$ with base $(\Omega, \mathcal{F}, P)$ but $\mathcal{P}^{1}$ does not have the countable concatenation property. Let $\mathcal{P}^{2}=\mathcal{P}^{1} \cup\{|\cdot|\}$, then $\mathcal{P}^{2}$ has the countable concatenation property and $\left(S, \mathcal{P}^{2}\right)$ is exactly the random normed module $\left(L^{0}(\mathcal{F}, R),|\cdot|\right)$. Define the mapping $T:\left(S, \mathcal{P}^{1}\right) \rightarrow\left(S, \mathcal{P}^{2}\right)$ by

$$
T x=\sum_{n=1}^{\infty} \tilde{I}_{A_{n}} \cdot \xi_{n} \cdot x, \forall x \in S,
$$

where $\xi_{n} \in L_{++}^{0}(\mathcal{F})$, then $T$ is a module homomorphism, and $T \in B\left(S_{\varepsilon, \lambda}^{1}, S_{\varepsilon, \lambda}^{2}\right)$ but $T \notin B\left(S_{c}^{1}, S_{c}^{2}\right)$. In fact, take $\xi=\sum_{i=1}^{\infty} \tilde{I}_{A_{n}} \cdot \xi_{n}, \varepsilon \in L_{++}^{0}(\mathcal{F})$, and observe that

$$
T^{-1}\{y \in S|| y \mid \leq \varepsilon\}=\left\{x \in S|| \xi^{-1} \cdot x \mid \leq \varepsilon\right\}
$$


then it is easy to see that $T^{-1}\{y \in S|| y \mid \leq \varepsilon\}$ is a neighborhood of $\theta \in S$ in the $(\varepsilon, \lambda)$-topology induced by $\mathcal{P}^{2}$, however, it is not a neighborhood of $\theta \in S$ in the locally $L^{0}$-convex topology induced by $\mathcal{P}^{1}$ since $|\cdot| \notin \mathcal{P}^{1}$.

\subsection{The relation between $B\left(S_{\varepsilon, \lambda}^{1}, S_{\varepsilon, \lambda}^{2}\right)$ and $B\left(S_{c}^{1}, S_{\varepsilon, \lambda}^{2}\right)$}

From the proof of Theorem 1.2 in [13], we can easily obtain Lemma 3.3 below, which plays an important role in the proof of Theorem 1.6.

Lemma 3.3. Let $\left(S^{1}, \mathcal{P}^{1}\right)$ be a random locally convex module over $K$ with base $(\Omega, \mathcal{F}, P)$ and $f$ a mapping from $S^{1}$ to $L_{+}^{0}(\mathcal{F})$ such that $f(\xi \cdot x)=\xi \cdot f(x)$ and $f(x+y) \leq f(x)+f(y), \forall \xi \in L_{+}^{0}(\mathcal{F})$ and $x, y \in S^{1}$. If $f:\left(S^{1}, \mathcal{T}_{c}^{1}\right) \rightarrow$ $\left(L^{0}(\mathcal{F}, K), \mathcal{T}_{\varepsilon, \lambda}\right)$ is continuous, then there exist a countable partition $\left\{A_{n}, n \in\right.$ $N\}$ of $\Omega$ to $\mathcal{F}$, a sequence $\left\{\xi_{n}, n \in N\right\}$ in $L_{+}^{0}(\mathcal{F})$ and a countable subset $\left\{\mathcal{Q}_{n}^{1}, n \in N\right\}$ in $\mathcal{F}\left(\mathcal{P}^{1}\right)$ such that

$$
f(x) \leq \sum_{n=1}^{\infty} \tilde{I}_{A_{n}} \cdot \xi_{n} \cdot\|x\|_{\mathcal{Q}_{n}^{1}}, \forall x \in S^{1} .
$$

We can now give the proof of Theorem 1.6.

Proof of Theorem 1.6. For each $\mathcal{Q}^{2} \in \mathcal{F}\left(\mathcal{P}^{2}\right)$, define the mapping $f_{\mathcal{Q}^{2}}: S^{1} \rightarrow$ $L_{+}^{0}(\mathcal{F})$ by

$$
f_{\mathcal{Q}^{2}}(x)=\|T x\|_{\mathcal{Q}^{2}}, \forall x \in S^{1},
$$

then $f_{\mathcal{Q}^{2}}(\xi \cdot x)=\xi \cdot f_{\mathcal{Q}^{2}}(x)$ and $f_{\mathcal{Q}^{2}}(x+y) \leq f_{\mathcal{Q}^{2}}(x)+f_{\mathcal{Q}^{2}}(y), \forall \xi \in L_{+}^{0}(\mathcal{F})$ and $x, y \in S^{1}$. If $T \in B\left(S_{c}^{1}, S_{\varepsilon, \lambda}^{2}\right)$, then it is easy to see that $f_{\mathcal{Q}^{2}}$ is a continuous mapping from $\left(S^{1}, \mathcal{T}_{c}^{1}\right)$ to $\left(L^{0}(\mathcal{F}, K), \mathcal{T}_{\varepsilon, \lambda}\right)$. Thus it follows from Lemma 3.3 that there exist a countable partition $\left\{A_{n}, n \in N\right\}$ of $\Omega$ to $\mathcal{F}$, a sequence $\left\{\xi_{n}, n \in N\right\}$ in $L_{+}^{0}(\mathcal{F})$ and a countable subset $\left\{\mathcal{Q}_{n}^{1}, n \in N\right\}$ in $\mathcal{F}\left(\mathcal{P}^{1}\right)$ such that

$$
\|T x\|_{\mathcal{Q}^{2}}=f_{\mathcal{Q}^{2}}(x) \leq \sum_{n=1}^{\infty} \tilde{I}_{A_{n}} \cdot \xi_{n} \cdot\|x\|_{\mathcal{Q}_{n}^{1}}, \forall x \in S^{1} .
$$

Since $T$ is random linear, it follows from Proposition 3.3 that $T \in B\left(S_{\varepsilon, \lambda}^{1}, S_{\varepsilon, \lambda}^{2}\right)$.

Conversely, it is clear.

\section{References}

[1] N. Dunford and J. T. Schwartz, Linear Operators, Part I, A Wiley-Interscience Publication. John Wiley \& Sons, Inc., New York, 1988.

[2] D. Filipović, M. Kupper, and N. Vogelpoth, Separation and duality in locally $L^{0}$-convex modules, J. Funct. Anal. 256 (2009), no. 12, 3996-4029.

[3] T. X. Guo, Extension theorems of continuous random linear operators on random domains, J. Math. Anal. Appl. 193 (1995), no. 1, 15-27.

[4] _ Module homomorphisms on random normed modules, Northeast. Math. J. 12 (1996), no. $1,102-114$.

[5] - Some basic theories of random normed linear spaces and random inner product spaces, Acta Anal. Funct. Appl. 1 (1999), no. 2, 160-184. 
[6] Survey of recent developments of random metric theory and its applications in China. II, Acta Anal. Funct. Appl. 3 (2001), no. 3, 208-230.

[7] _ The relation of Banach-Alaoglu theorem and Banach-Bourbaki-KakutaniŚmulian theorem in complete random normed modules to stratification structure, Sci. China Ser. A 51 (2008), no. 9, 1651-1663.

[8] _ Relations between some basic results derived from two kinds of topologies for a random locally convex module, J. Funct. Anal. 258 (2010), no. 9, 3024-3047.

[9] _ Recent progress in random metric theory and its applications to conditional risk measures, Sci. China Math. 54 (2011), no. 4, 633-660.

[10] T. X. Guo and S. B. Li, The James theorem in complete random normed modules, J. Math. Anal. Appl. 308 (2005), no. 1, 257-265.

[11] T. X. Guo and G. Shi, The algebraic structure of finitely generated $L^{0}(\mathcal{F}, K)$-modules and the Helly theorem in random normed modules, J. Math. Anal. Appl. 381 (2011), no. $2,833-842$.

[12] T. X. Guo and X. Zhang, Stone's representation theorem of a group of random unitary operators on complete complex random inner product modules (in Chinese), Sci. Sin. Math. 42 (2012), no. 3, 181-202.

[13] T. X. Guo and S. E. Zhao, On the random conjugate spaces of a random locally convex module, Acta Math. Sin. (Engl. Ser.) 28 (2012), no. 4, 687-996.

[14] T. X. Guo, S. E. Zhao, and X. L. Zeng, On random convex analysis-the analytic foundation of the module approach to conditional risk measures, arXiv:1210.1848, (2012).

[15] T. X. Guo and L. H. Zhu, A characterization of continuous module homomorphisms on random semi-normed modules and its applications, Acta Math. Sin. (Engl. Ser.) 19 (2003), no. 1, 201-208.

[16] B. Schweizer and A. Sklar, Probabilistic Metric Spaces, Elsevier/North-Holland, New York, 1983; Dover Publications, New York, 2005.

[17] M. Z. Wu, The Bishops-Phelps theorem in complete random normed modules endowed with the $(\varepsilon, \lambda)$-topology, J. Math. Anal. Appl. 391 (2012), no. 2, 648-952.

[18] _ A further study on the Riemann-intergrability for abstract-valued functions from a closed real interval to a complete random normed module (in Chinese), Sci. Sin. Math. 42 (2012), no. 9, 897-903.

[19] X. Zhang, On mean ergodic semigroups of random linear operators, Proc. Japan Acad. Ser. A Math. Sci. 88 (2012), no. 4, 53-58.

[20] - On conditional mean ergodic semigroups of random linear operators, J. Inequal. Appl. 150 (2012), 1-10.

[21] X. Zhang and T. X. Guo, Von Neumann's mean ergodic theorem on complete random inner product modules, Front. Math. China 6 (2011), no. 5, 965-985.

[22] S. E. Zhao and T. X. Guo, The random subreflexivity of complete random normed modules, Internat. J. Math. 23 (2012), no. 3, 1-14

[23] S. E. Zhao and G. Shi, A geometric form of the Hahn-Banach extension theorem for $L^{0}$ linear functions and the Goldstine-Weston theorem in random normed modules (in Chinese), Sci. Sin. Math. 41 (2011), no. 9, 827-836.

Department of Mathematics

SCHOOL OF SCIENCE

Tianjin POLYTEChNiC University

Tianjin 300387, P. R. China

E-mail address: xiazhangxz@gmail.com 